\title{
O USO DO DISPOSITIVO INTRAUTERINO (DIU) EM MULHERES
}

\section{Munah Najeh Saleh Ahmad Maruf1; Gicelle Moraes Martelli²; Ana Luiza Rios Antunes 3 ; Eduarda Rodrigues Machado4; Eduardo Marques Machado5; Luiza Fortes Lamberty6; Flávia Dorneles Saleh ${ }^{7}$; Claudia Maria Gabert Diaz ${ }^{8}$.}

\section{RESUMO}

Objetivo: enfatizar a importância e os direitos das mulheres referente ao uso do Dispositivo Intrauterino (DIU), evidenciando os prós e contras deste método contraceptivo. Com direcionamento à mulheres que buscam um contraceptivo sem presença de hormônios, com alta resolutividade, longa duração e fácil reversão. Metodologia: estudo de natureza teórico-reflexivo, cuja fundamentação baseia-se na formulação discursiva acerca da temática. Consultado em materiais acadêmicos científicos. Resultados e Dsicussões: o DIU de Cobre mostrou resultados satisfatórios em mulheres que possuem contraindicação de estrogênio, que estão na perimenopausa e em lactantes, pelo fato de não possuir hormônios. O DIU Hormonal Mirena não é disponibilizado pelo Sistema Único de Saúde no Brasil, porém encontra-se disponível em clínicas particulares e convênios de saúde. Conclusão: conclui-se que é altamente eficaz como método de anticoncepção, tratamento de doenças e também como uma forma de inserção simples.

Palavras-chave: Autonomia; Liberdade de Escolha; Métodos Contraceptivos.

\begin{abstract}
Objective: to emphasize the importance and rights of women regarding the use of the IUD, highlighting the pros and cons of this contraceptive method. Directed to women who seek a method without the presence of hormones, with high resolution, long duration and easy reversal. Methodology: theoretical-reflective study, whose foundation is based on the discursive formulation about the theme. Consulted in scientific academic materials. Results and Discussion: The Copper IUD has shown

\footnotetext{
${ }_{1}^{1}$ Munah Najeh Saleh Ahmad Maruf - Universidade Franciscana - UFN, munahsaleh1999@gmail.com.

2 Gicelle Moraes Martelli - Universidade Franciscana - UFN, gicelle1219@gmail.com

${ }^{3}$ Ana Luiza Rios Antunes - Universidade Franciscana - UFN, riosana1306@gmail.com

${ }^{4}$ Eduarda Rodrigues Machado - Universidade Franciscana - UFN, eduardamachado886@gmail.com

5 Eduardo Marques Machado - Universidade Franciscana - UFN, eduardomarques051@gmail.com

6 Luiza Fortes Lamberty - Universidade Franciscana - UFN, luizaflamberty@gmail.com

7 Flávia Dorneles Saleh - Universidade Franciscana - UFN, flaviasaleh222@gmail.com

${ }^{8}$ Claudia Maria Gabert Diaz - Universidade Franciscana - UFN, cmgdiaz12@gmail.com
} 
satisfactory results in women who have contraindications for estrogen, who are in perimenopause and in breastfeeding, because they do not have hormones. The Hormonal Mirena IUD is not provided by the Unified Health System in Brazil, but it is available in private clinics and health plans. Conclusion: it is concluded that this is highly effective as a method of contraception, treatment of diseases and also as a form of simple insertion.

Key-words: Autonomy; Freedom of choice; Contraceptive Methods.

\section{INTRODUÇÃO}

A proposta inicial para a inserção do uso do Dispositivo Intrauterino (DIU) no Sistema Único de Saúde (SUS) foi dada através de ações para tentar reduzir a taxa de mortalidade materna no Brasil. Através de pesquisas, foi evidenciado que mais da metade da população entrevistada não havia planejado a gestação. Pondo em pauta estes dados, foi tomada a iniciativa de adicionar este método contraceptivo, a fim de permitir o direito da família decidir e planejar o momento de ter uma criança em suas vidas (BRASIL, 2018).

A lei $n^{\circ} 9263$ de 12 de janeiro de 1996 regulamenta o $\S 7^{\circ}$ do art. 226 da Constituição Federal, que trata do Planejamento Familiar, e determina que, para o seu exercício, devem ser oferecidos todos os métodos e técnicas de concepção e contracepção cientificamente aceitos, que não coloquem em risco a vida e a saúde das pessoas, garantindo a liberdade de opção (BRASIL, 2018).

Mulheres dentro do período de vida reprodutiva, podem escolher qual o método contraceptivo que considera melhor as suas singularidades. O DIU se tornou uma opção bastante procurada e utilizada principalmente no sistema de saúde pública. A facilidade e a tranquilidade que o método oferece, fez com que muitas deixassem de lado a utilização da pílula anticoncepcional.

No Brasil, é disponibilizado pelo SUS o DIU de Cobre TCu 380, o mesmo é constituído por um pequeno e flexível dispositivo de polietileno em formato de $\mathrm{T}$, revestido com $314 \mathrm{~mm} 2$ de cobre na haste vertical e dois anéis de $33 \mathrm{~mm} 2$ de cobre em cada haste horizontal (BRASIL, 2018).

Outro modelo de dispositivo bastante utilizado é o Diu Hormonal Mirena, é um pequeno dispositivo com formato de $\mathrm{T}$, possui um cilindro com liberação lenta de 
Levanorgestrel, semelhante à progesterona feminina, e um fio na extremidade distal que fica aparente pelo colo uterino. Além de servir como anticoncepcional, possui função para o tratamento de algumas doenças que acometem mulheres (MONTEIRO, 2015).

O DIU de Cobre é um método contraceptivo do grupo dos LARCs. Na região interna do útero, exerce funções locais que evitam a gestação, apresentando-se como um dos métodos mais seguros para evitar uma gravidez não planejada. Não possui a necessidade de intervenções diárias da mulher e não prejudica a fertilidade futura. O procedimento da inserção dura em torno de 10 minutos (BRASIL, 2018).

Este dispositivo não contém nenhuma composição hormonal, é altamente efetivo (mais de 99\%) e possui um custo-benefício satisfatório. Possui duração de 10 anos e um retorno quase imediato à fertilidade, sem efeitos sistêmicos e não interfere na lactação (BRASIL, 2018).

Seu mecanismo de ação age estimulando mudanças bioquímicas e morfológicas no endométrio conforme os íons são liberados no útero, ocasionando uma ação inflamatória e citotóxica com efeito espermicida. O dispositivo é responsável pelo aumento da produção de prostaglandinas e pela inibição de enzimas endometriais. Com isso, é ocasionado efeitos nos espermatozoides e ovócitos.

\section{METODOLOGIA}

Este respectivo estudo é de natureza teórico-reflexivo, cuja fundamentação baseia-se na formulação discursiva acerca da temática. Consultado em materiais acadêmicos científicos, onde os mesmos foram relativos ao uso do Dispositivo Intrauterino de Cobre e Hormonal. Mostrando e evidenciando aspectos importantes e relevantes à saúde e planejamento da vida da mulher.

\section{RESULTADOS E DISCUSSÕES}

Considera-se que o DIU interfere na motilidade e qualidade do espermatozoide, atrapalhando a ascensão dos mesmos, desde a vagina até as 
tubas uterinas, ocasionando a morte destes pelo aumento na produção de citocinas citotóxicas (BRASIL, 2018).

É indicado especialmente a mulheres que buscam um método contraceptivo sem a presença de hormônios, com alta resolutividade, com longa duração e com fácil reversão. As informações podem ser ofertadas em momentos de educação em saúde com as usuárias, podendo ser realizadas na Atenção básica, visitas domiciliares e em clínicas.

O DIU de Cobre mostrou resultados muito satisfatórios em mulheres que possuem contraindicação de estrogênio, que estão na perimenopausa e em mulheres que estão amamentando. Visto que não possui hormônios, é muito efetivo durante o período da lactação, pois não ocasiona alterações na quantidade e qualidade do leite materno. Como efeitos colaterais, destaca-se o aumento do fluxo sanguíneo durante o período menstrual e cefaleias intensas (BRASIL, 2018).

Para mulheres com menos de 50 anos, recomenda-se retirá-lo dois anos após a última menstruação. Quando obtiver mais de 50 anos, recomenda-se esperar um ano de amenorreia para a sua retirada. Porém, não há problemas se por alguma razão, a mulher manter o DIU inserido após a menopausa (BRASIL, 2018).

Possui contraindicação em casos de IST's (infecções sexualmente transmissíveis), tais como clamídia, gonorreia e AIDS nos estágios clínicos 3 e 4. Em usuárias positivadas para sífilis (já tratadas) e HIV assintomáticas, não há contraindicação para o uso do DIU. Presença de infecção inflamatória pélvica aguda ou crônica, endometrite, cervicite mucopurulenta e tuberculose pélvica contraindicam a inserção. Anormalidades uterinas como útero bicorno, septado ou intensa estenose cervical impedem o uso do mesmo também (BRASIL, 2018).

O DIU Hormonal Mirena não é disponibilizado pelo Sistema Único de Saúde no Brasil, porém encontra-se disponível em clínicas particulares e convênios de saúde. Possui duração de 5 anos e libera diariamente $20 \mu \mathrm{g}$ de Levonorgestrel (LNG), um progestógeno (MONTEIRO, 2015).

Serve tanto como método de anticoncepção, como também para tratamento de algumas doenças femininas, tais como, endometriose e hemorragias disfuncionais. Possui 99\% de eficácia e é altamente seguro e confortável para as 
mulheres que fazem o uso. Para voltar a fertilidade, o processo pode ocorrer um pouco mais lentamente, levando até 3 meses para retornar, por conta da questão hormonal (MACHADO, 2018).

Sobre os efeitos colaterais, apesar de ter um efeito local da liberação deste hormônio, a progesterona, há absorção sistêmica principalmente nos primeiros meses de uso. Dentre os sintomas, pode ocorrer de sentir cefaleia, edemas, acne, dor na região abdominal, perda de cabelo, dor pélvica e sangramento disfuncional. É contraindicado para quem teve câncer de mama nos últimos 5 anos, ou mulheres que possuem doenças hepáticas, devem evitá-lo e optar por outro tipo de método (MONTEIRO, 2015).

Para a inserção tanto do DIU de Cobre, quanto ao Mirena, pode ser inserido em qualquer dia do ciclo menstrual, desde que se tenha certeza de que a usuária não esteja grávida, no pós-parto ou pós-abortamento imediatos. Recomenda-se exame ginecológico completo antes da inserção, para que se possa avaliar o conteúdo vaginal, posição e volume uterino (MACHADO, 2018).

A colocação do Dispositivo Intrauterino, deve ser realizada por um médico ou enfermeiro após um treinamento. Desta maneira, a mulher deve passar por uma consulta prévia, por uma anamnese, visando esclarecer as dúvidas, mecanismos de ação e as condições para o uso (MACHADO, 2018).

Vale atentar que, a utilização destes métodos, não impede que as mulheres adquiram ou transmitam doenças sexualmente transmissíveis. Infelizmente, ao utilizar outros métodos, a grande maioria deixa de utilizar preservativos e esquecem da importância que ele tem para a sua saúde.

É imprescindível que os profissionais saibam o que estão fazendo e como deve ser a colocação corretamente, visto que, se mal colocado pode não só prejudicar a saúde da mulher, mas também diminuir a eficácia no controle de natalidade. Ademais, o uso do DIU de cobre se tornou uma grande opção, por ser um método sem presenças de hormônios e com um longo período de duração, além do custo-benefício.

Entretanto, o aumento do fluxo menstrual pode se tornar um motivo de arrependimento da colocação do DIU de cobre. Assim como o aumento ou 
aparecimento transitórios de cólicas menstruais. Muitas veem o DIU como um método abortivo, porém, o mesmo apenas impede o encontro entre o óvulo com o espermatozoide através das modificações morfológicas e bioquímicas (BRASIL, 2018).

\section{CONCLUSÃO}

Portanto, conclui-se que este é altamente eficaz como método de anticoncepção, tratamento de doenças e também como uma forma de inserção simples. O qual não precisa de manutenção por um longo período de tempo, confortável e respeitando a autonomia, escolha e singularidade de cada mulher.

O incremento de ações que visam a divulgação de métodos contraceptivos na Atenção Primária em Saúde, são fundamentais não só para a valorização da saúde das mulheres, mas também na prevenção de gravidez indesejada. As divulgações devem ser de maneira qualificada e por profissionais que entendam as especificidades de cada método contraceptivo. Visto que, o Dispositivo Intrauterino, é rodeado por evidências científicas, assim como todos os outros métodos.

\section{AGRADECIMENTOS}

Agradeço primeiramente as minhas colegas e orientadora que junto a mim realizaram este trabalho, e a Universidade Franciscana pela oportunidade e incentivo a pesquisa.

\section{REFERÊNCIAS}

BRASIL. Ministério da Saúde. Secretaria de Atenção à Saúde. MANUAL TÉCNICO PARA PROFISSIONAIS DE SAÚDE - DIU COM COBRE TCu 380 A. Brasília, DF, 2018. Disponível em: http://portaldeboaspraticas.fff.fiocruz.br/wpcontent/uploads/2018/12/manual diu 08 2018.pdf.

MACHADO, R.B. Uso de dispositivos intrauterinos (DIU) em nulíparas. São Paulo: Federação das Associações Brasileiras de Ginecologia e Obstetrícia (FEBRASGO); $2018 . \quad 13 \quad$ p. $\quad$ Disponível em: https://www.febrasgo.org.br/media/k2/attachments/16-serie diu.pdf. 
EDUCAÇÃO, SAÚDE

ETECNOLOGIA

26 A 28 DE OUTUBRO DE 2021

\section{QUFN}

MONTEIRO, I.M.U. Contracepção de longo prazo: dispositivo intrauterino (Mirena).

Rev.Femina. Campinas, v. 43 n. 1 p. 22-60, fevereiro, 2015. Disponível em: http://files.bvs.br/upload/S/0100-7254/2015/v43nsuppl1/a4851.pdf. 